\title{
On the infinite-dimensional moment problem
}

\author{
Konrad Schmüdgen
}

\begin{abstract}
This paper deals with the moment problem on a (not necessarily finitely generated) commutative unital real algebra $A$. We define moment functionals on $A$ as linear functionals which can be written as integrals over characters of $A$ with respect to cylinder measures. Our main results provide such integral representations for $A_{+}$-positive linear functionals (generalized Haviland theorem) and for positive functionals fulfilling Carleman conditions. As an application, we solve the moment problem for the symmetric algebra $S(V)$ of a real vector space $V$. As a byproduct, we obtain new approaches to the moment problem on $S(V)$ for a nuclear space $V$ and to the integral decomposition of continuous positive functionals on a barrelled nuclear topological algebra $A$.
\end{abstract}

\section{Introduction}

The moment problem on $\mathbb{R}^{d}$ is a well-studied classical research topic, see e.g. [2], [7], [27]. In modern formulation, it is the following question: When can a linear functional on the polynomial algebra $\mathbb{R}\left[x_{1}, \ldots, x_{d}\right]$ (or on a finitely generated commutative real algebra $A$ ) be expressed as an integral over point evaluations with respect to some Radon measure on $\mathbb{R}^{d}$ (or on the character space $\hat{A}$ )?

This paper is about the moment problem in infinitely many variables. Suppose that $A$ is a (not necessarily finitely generated) commutative unital real algebra. We study the following generalization of the classical moment problem:

When is a linear functional $L$ on $A$ an integral over characters of $A$ ?

If $A$ is finitely generated, Haviland's theorem [27, Theorem 1.14] provides an answer: it holds if and only if $L$ is $A_{+}$-positive, that is, $L$ is nonnegative on elements of $A$ that are nonnegative on all characters. Note that the latter condition is difficult to

Key words and phrases: moment problem, cylinder measure, symmetric algebra, nuclear space, Carleman condition.

2010 Mathematics Subject Classification: primary 44A60; secondary 46G12, 28C20. 
verify in general. Further, if $L$ is only nonnegative on squares and satisfies Carleman growth conditions, then it is a moment functional [27, Theorem 14.20]. The aim of the present paper is to generalize these two fundamental results to not necessarily finitely generated algebras.

From the conceptional side, our new contribution is that we do not define moment functionals by integrals with respect to Radon measures, but only as integrals over cylinder measures. This allows us to solve moment problems for more general algebras without nuclearity assumptions.

Our main result in this respect states that under corresponding natural assumptions a linear functional $L$ on $A$ is an integral of characters over a cylinder measure.

The next natural question is: When is $L$ an integral of some ( $\sigma$-additive) measure? From Minlos' theorem it follows that this holds if some nuclearity assumption is added. As an immediate consequence we obtain new and unifying approaches for two fundamental results: the moment problem for symmetric algebras over nuclear spaces (Theorem 16) and the decomposition of continuous positive functionals on barrelled nuclear topological algebras as integrals over characters (Theorem 15). Note that for both results the known proofs contained in the literature are quite involved and based on different techniques.

This paper is organized as follows. Section 2 reviews basic definitions and results on cylinder measures. In Section 3 we define moment functionals with respect to cylinder measures. Section 4 contains the main results of this paper. We prove an integral representation theorem (Theorem 12) with respect to cylinder measures for $A_{+}$-positive functionals and for positive functionals satisfying Carleman conditions. In Section 5 we assume that the underlying space is nuclear. Then, combined with Minlos's theorem, we obtain integral representations by "ordinary" $\sigma$-additive measures (Theorems 13 and 15). In Section 6 we treat the moment problem for the symmetric algebra $S(V)$ of a real vector space $V$ (Theorem 16). Section 7 deals with moment functionals with respect to Gaussian cylinder measures. We give examples which have representing measures and examples for which there are only representing cylinder measures, but no representing measures.

All results in this paper are developed for a real commutative algebra $A$, but they are easily extended to complex commutative *-algebras by passing to the complexification $A_{\mathbb{C}}$ of $A$. Recall that the direct sum $A_{\mathbb{C}}:=A \oplus \mathrm{i} A$ of vector spaces $A$ and i $A$ becomes a complex commutative $*$-algebra, called the complexification of $A$, with multiplication, involution and scalar multiplication defined by

$$
\begin{gathered}
(a+\mathrm{i} b)(c+\mathrm{i} d)=a c-b d+\mathrm{i}(b c+a d),(a+\mathrm{i} b)^{*}:=a-\mathrm{i} b, \\
(\alpha+\mathrm{i} \beta)(a+\mathrm{i} b):=\alpha a-\beta b+\mathrm{i}(\alpha b+\beta a),
\end{gathered}
$$


where $a, b, c, d \in A$ and $\alpha, \beta \in \mathbb{R}$. Each $\mathbb{R}$-linear functional $L: A \rightarrow \mathbb{R}$ extends uniquely to a $\mathbb{C}$-linear functional $L_{\mathbb{C}}: A_{\mathbb{C}} \rightarrow \mathbb{C}$ by $L_{\mathbb{C}}(a+\mathrm{i} b)=L(a)+\mathrm{i} L(b), a, b \in A$. Clearly, $L\left(a^{2}\right) \geq 0$ for all $a \in A$ if and only if $L_{\mathbb{C}}\left(x^{*} x\right) \geq 0$ for all $x \in A_{\mathbb{C}}$.

Infinite-dimensional moment problems were first studied by A. G. Kostyuchenko and B.S. Mityagin [18], Y. M. Berezansky and S.N. Sifrin [6], H.J. Borchers and J. Yngvason [9] for symmetric algebras over nuclear spaces and since then by various authors, see [1], [3], [5], [10], [11], [14], [15], [17], [19], [23], [28]. Infinite-dimensional versions of the moment theorem for compact semi-algebraic sets [24] are obtained in [1], [3].

\section{Cylinder measures}

In this section, we recall some basics on cylinder measures; our standard reference is [16, Chapter IV].

Suppose that $V$ is a real locally convex Hausdorff space. Let $V^{\prime}$ denote the vector space of all continuous real-valued linear functionals on $V$.

Suppose that $\mathcal{F}$ is a finite-dimensional linear subspace of $V$. Its annihilator space $\mathcal{F}^{\circ}$ is defined by

$$
\mathcal{F}^{\circ}=\left\{L \in V^{\prime}: L(f)=0 \text { for } f \in \mathcal{F}\right\} .
$$

The quotient space $V^{\prime} / \mathcal{F}^{\circ}$ is also finite-dimensional. If $M$ is a Borel subset of $V^{\prime} / \mathcal{F}^{\circ}$, then the set $N$ of all $L \in V^{\prime}$ which are mapped into elements of $M$ under the canonical mapping of $V^{\prime}$ into $V^{\prime} / \mathcal{F}^{\circ}$ is called the cylinder set with base $M$ and generating subspace $V^{\prime} / \mathcal{F}^{\circ}$. The cylinder sets form an algebra of sets denoted by $\mathcal{C}\left(V^{\prime}\right)$. Let $\mathcal{B}\left(V^{\prime}\right)$ denote the $\sigma$-algebra generated by all cylinder sets. Clearly, $\mathcal{B}\left(V^{\prime}\right)$ is the Borel $\sigma$-algebra when the vector space $V^{\prime}$ is equipped with the weak topology $\sigma\left(V^{\prime}, V\right)$.

From [16, Chapter IV, §1,3.] we restate the following definition.

Definition 1. A cylinder measure on $V^{\prime}$ is a real-valued function $\mu$ on the algebra $\mathcal{C}(V)$ such that:

(i) $0 \leq \mu(N) \leq 1$ for all $N \in \mathcal{C}(V)$.

(ii) $\mu\left(V^{\prime}\right)=1$.

(iii) If $N$ is the union of pairwise disjoint sets $N_{n} \in \mathcal{C}(V), n \in \mathbb{N}$, with Borel base sets and a common generating subspace $V^{\prime} / \mathcal{F}^{\circ}$, then

$$
\mu(N)=\sum_{n=1}^{\infty} \mu\left(N_{n}\right) .
$$

In [16] there is a fourth condition, but it follows from the three others. 
Definition 2. A cylinder measure $\mu$ on $V^{\prime}$ is called continuous if for each $\varepsilon>0$ and $a>0$ there exists a neighbourhood of zero, $U$, in $V$ such that for all $t \in U$ we have

$$
\mu\left(\left\{\chi \in V^{\prime}:|\chi(t)| \geq a\right\}\right) \leq \varepsilon .
$$

This definition of continuity is equivalent to the definition given in [16], as noted in [16, Ch. IV, §1, 4.] and proved in [20]. It is easily seen that we may restrict ourselves to the case when $a=1$.

Now suppose that $\mu$ is a cylinder measure on $V^{\prime}$. Then $\mu$ defines a normalized measure $\nu_{\mathcal{F}}$ on the Borel $\sigma$-algebra of each factor space $V^{\prime} / \mathcal{F}^{\circ}$ by

$$
\nu_{\mathcal{F}}(M):=\mu(N)
$$

where $N$ is the cylinder set with base $M$ and generating space $V^{\prime} / \mathcal{F}^{\circ}$. These measures $\nu_{\mathcal{F}}$ are not independent of each others. If $\mathcal{G}$ is another finite-dimensional subspace of $V^{\prime}$ such that $\mathcal{F} \subseteq \mathcal{G}$, then for each Borel set $M$ of $V^{\prime} / \mathcal{F}^{\circ}$ we have

$$
\nu_{\mathcal{F}}(M)=\nu_{\mathcal{G}}\left(\pi^{-1}(M)\right)
$$

where $\pi$ denotes the canonical map of $V^{\prime} / \mathcal{G}^{\circ}$ into $V^{\prime} / \mathcal{F}^{\circ}$ given by $\pi\left(L+\mathcal{G}^{\circ}\right)=L+\mathcal{F}^{\circ}$, $L \in V^{\prime}$. Note that (3) is a compatibility condition for the measures $\nu_{\mathcal{F}}$. That is, if (3) holds for a family of normalized measures $\nu_{\mathcal{F}}$ indexed by the finite-dimensional subspaces $\mathcal{F}$ of $V$, then it comes from a cylinder measure $\mu$, defined by (2), on $V^{\prime}$ (see e.g. [16, Chapter IV, §1, 5.].

Cylinder measures are defined only on the algebra $\mathcal{C}(V)$ of cylinder sets and they are not $\sigma$-additive in general. Hence it is natural to look for conditions on $V$ which imply a cylinder measure $\mu$ on $V^{\prime}$ is $\sigma$-additive on $\mathcal{C}(V)$. In this case $\mu$ extends to an "ordinary" $\sigma$-additive measure on the $\sigma$-algebra $\mathcal{B}(V)$.

In what follows, nuclear operators and nuclear spaces will play a crucial role. Definitions and basic facts on these notions can be found (for instance) in [16, Chapter I, §3] or [22, Chapter III, 7.]. We briefly repeat some of them.

Let $\left(V_{1},\langle\cdot, \cdot\rangle_{1}\right)$ and $\left(V_{2},\langle\cdot, \cdot\rangle_{2}\right)$ be Hilbert spaces. An operator $b: V_{1} \rightarrow V_{2}$ is called nuclear if there exist sequences $\left(\varphi_{n}\right)_{n \in \mathbb{N}}$ of $V_{1}$ and $\left(\psi_{n}\right)_{n \in \mathbb{N}}$ of $V_{2}$ such that

$$
\sum_{n=1}^{\infty}\left\|\varphi_{n}\right\|_{1}\left\|\psi_{n}\right\|_{2}<\infty \quad \text { and } \quad b=\sum_{n=1}^{\infty}\left\langle\cdot, \varphi_{n}\right\rangle_{1} \psi_{n} .
$$

A positive self-adjoint operator $b: V \rightarrow V$ of a Hilbert space $(V,\langle\cdot, \cdot\rangle)$ is nuclear, equivalently of trace class, if and only if $\sum_{i \in I}\left\langle b \eta_{i}, \eta_{i}\right\rangle<\infty$ for some (then for each) orthonormal basis $\left(\eta_{i}\right)_{i \in I}$ of $V$. 
Let $\left(V_{n},\langle\cdot, \cdot\rangle_{n}\right), n \in \mathbb{N}$, be Hilbert spaces such that $V_{n} \subseteq V_{m}$ and $\|\cdot\|_{m} \leq\|\cdot\|_{n}$ for all $n \geq m$. The vector space $V=\cap_{n} V_{n}$, equipped with the locally convex topology defined by the family of norms $\|\cdot\|_{n}, n \in \mathbb{N}$, is called a $\sigma$-Hilbert space. Such a space $V$ is called nuclear if for each $n \in \mathbb{N}$ there exists an $m \in \mathbb{N}$ such that the embedding of the Hilbert space $\left(V_{m},\langle\cdot, \cdot\rangle_{m}\right)$ into $\left(V_{n},\langle\cdot, \cdot\rangle_{n}\right)$ is nuclear.

Note that the Schwartz space $\mathcal{S}\left(\mathbb{R}^{d}\right)$ is a nuclear $\sigma$-Hilbert space. Indeed, let $N$ denote the number operator with domain $\mathcal{D}(N)$ in the Hilbert space $\left(L^{2}\left(\mathbb{R}^{d}\right),\langle\cdot, \cdot\rangle\right)$. Then $\mathcal{S}\left(\mathbb{R}^{d}\right)=\cap_{n} V_{n}$, where $V_{n}:=\mathcal{D}\left(N^{n}\right)$ and $\langle\cdot, \cdot\rangle_{n}:=\left\langle N^{n} \cdot, N^{n} \cdot\right\rangle, n \in \mathbb{N}$.

An important and deep result is the following Minlos' theorem.

Theorem 3. Suppose that $V$ is a nuclear locally convex Hausdorff space. Then each continuous cylinder measure on $V^{\prime}$ is $\sigma$-additive and extends to a measure on the $\sigma$-algebra $\mathcal{B}(V)$.

Proof. If $V$ is a $\sigma$-Hilbert space, this result was proved by R.A. Minlos [20]. A proof of this case is given in [16, pp. 290-30]. The general case is treated in [29].

Now we specialize to the case when $V$ is a Hilbert space with scalar product $\langle\cdot, \cdot\rangle$. Let $\left(b_{n}\right)_{n \in \mathbb{N}}$ be a sequence of positive nuclear operators on $V$. Then the sets

$$
U_{n, r}:=\left\{t \in V:\left\langle b_{n} t, t\right\rangle \leq r\right\}, \quad \text { where } n \in \mathbb{N}, r>0,
$$

form a 0 -neighborhood basis of a locally convex topology on $V$, called the Sazonov topology associated with the sequence $\left(b_{n}\right)_{n \in \mathbb{N}}$.

A cylinder measure $\mu$ on $V^{\prime}$ is called continuous with respect to the Sazonov topology if for each $\varepsilon>0$ there exist $n \in \mathbb{N}$ and $\delta>0$ such that $\left\langle b_{n} t, t\right\rangle \leq \delta$ implies that $\mu(\{s \in V: \mid\langle s, t\rangle \geq 1\}) \leq \varepsilon$.

The following Sazonov theorem characterizes $\sigma$-additive cylinder measures.

Theorem 4. Suppose that $V$ is a Hilbert space. A cylinder measure $\mu$ on $V^{\prime}$ is $\sigma$-additive if and only if there exists a sequence $\left(b_{n}\right)_{n \in \mathbb{N}}$ of positive nuclear operators on $V$ such that $\mu$ is continuous in the corresponding Sazanov topology.

Proof. [16, Chapter IV, §2, Proposition 7] or [21].

\section{Moment functionals with cylinder measures}

Let us introduce our setup and some notations that will be kept in this paper. Throughout, $A$ is a unital real commutative algebra. We suppose that $T$ is a linear subspace of $A$ such that $T \cup\{1\}$ generates the algebra $A$ and $S$ is a vector space basis of $T$. The vector space of all linear functionals on $T$ is denoted by $T^{*}$. 
By a character of $A$ we mean an algebra homomorphism $\chi: A \rightarrow \mathbb{R}$ such that $\chi(1)=1$. The set of characters of the commutative algebra $A$ is denoted by $\hat{A}$. A linear functional $L$ on $A$ is called positive if $L\left(f^{2}\right) \geq 0$ for all $f \in A$.

Obviously, each character of $A$ is uniquely determined by its values on $T$. Hence the map $\hat{A} \ni \chi \rightarrow \chi\left\lceil T \in T^{*}\right.$ is injective. Simple examples show that this map is not surjective in general, that is, its range $\hat{A}\lceil T \equiv\{\chi\lceil T ; \chi \in \hat{A}\}$ can be different from $T^{*}$. For notational simplicity we will not distinguish between $\chi \in \hat{A}$ and its restriction $\chi\lceil T$. In fact, with a slight abuse of notation, we consider $\hat{A}\lceil T$ as a subset of $\hat{A}$. Further, we write $f(\chi):=\chi(f)$ for $f \in A, \chi \in \hat{A}$ and consider elements $f \in A$ as functions on $\hat{A}$.

Let $\mathcal{T}$ denote the vector space $T$ equipped with some locally convex Hausdorff topology. As usual, $\mathcal{T}^{\prime}$ is the dual vector space of all continuous linear functionals on $\mathcal{T}$. In what follows we will deal with cylinder measures on $\mathcal{T}^{\prime}$. (For instance, if we take the finest locally convex topology on the vector space $\mathcal{T}$, then $\mathcal{T}^{\prime}=T^{*}$.)

Definition 5. Suppose that $\mathcal{T}^{\prime} \subseteq \hat{A}\left\lceil T\right.$. Let $\mu$ be a cylinder measure on $\mathcal{T}^{\prime}$ such that the function $\chi \mapsto f(\chi)$ on $\mathcal{T}^{\prime}$ is $\mu$-integrable for all $f \in A$. The linear functional $L$ on $A$ defined by

$$
L(f)=\int_{\mathcal{T}^{\prime}} f(\chi) d \mu(\chi) \text { for } f \in A,
$$

is called a moment functional on $A$ and $\mu$ a representing cylinder measure of $L$.

Since $\mathcal{T}^{\prime} \subseteq \hat{A}\lceil T$, equation (5) means that $L$ is an integral over characters of $A$ with respect to the cylinder measure $\mu$.

The following example shows how the classical moment problem fits into the above setup.

Example 6. Let $A=\mathbb{R}\left[x_{1}, \ldots, x_{d}\right]$ and $T:=\left\{a_{1} x_{1}+\ldots+a_{d} x_{d}: a_{1}, \ldots, a_{d} \in \mathbb{R}\right\}$. Since $\hat{A}$ consists of point evaluations at points of $\mathbb{R}^{d}$, we have $\hat{A}\left\lceil T=T^{*}=\mathcal{T}^{\prime} \cong \mathbb{R}^{d}\right.$ for any locally convex Hausdorff topology on $T$. In this case, the moment functionals according to Definition 5 are just the "usual" moment functionals on $\mathbb{R}\left[x_{1}, \ldots, x_{d}\right]$.

All integral representations of functionals $L$ developed below remain valid without the assumption $\mathcal{T}^{\prime} \subseteq \hat{A}\lceil T$. The latter is only used to conclude that $L$ is an integral over characters of $A$, so that $L$ is a moment functional.

In the rest of this section, we discuss the condition $\mathcal{T}^{\prime} \subseteq \hat{A}\lceil T$ in Definition 5 .

If the character set $\hat{A}$ separates the points of $T$ (that is, $t \in T$ and $\chi(t)=0$ for all $\chi \in \hat{A}$ imply $t=0$ ), this assumption is fulfilled if we equip $T$ with the weak topology $\sigma(T, \hat{A}\lceil T)$ of the dual pairing $(T, \hat{A}\lceil T)$. This topology is Hausdorff by the separation assumption and we have $\mathcal{T}^{\prime}=\hat{A}\lceil T$ by a standard result on locally convex spaces. 
Now let us consider the case when $\hat{A}$ does not separate the points of $A$. Clearly,

$$
\operatorname{Rad}_{\mathrm{ch}}(A):=\{f \in A: \chi(f)=0 \text { for all } \chi \in \hat{A}\}
$$

is an ideal of $A$ and the quotient algebra $A / \operatorname{Rad}_{\mathrm{ch}}(A)$ has the property that the characters separate the points.

If $L$ is a positive functional on $A$ and $L$ vanishes on $f \in \operatorname{Rad}_{\text {ch }}(A)$, then $L$ induces a positive functional on the quotient algebra $A / \operatorname{Rad}_{\mathrm{ch}}(A)$ and we can study the moment problem there. Since this quotient algebra characters separate points, we can proceed as in the preceding remark.

The following question came up some years ago in a discussion with Y. Savchuk:

$\left(^{*}\right)$ Do all positive functionals on $A$ vanish on $\operatorname{Rad}_{\mathrm{ch}}(A)$ ?

For finitely generated algebras this follows easily from Haviland's theorem. As noted by T. Bisgaard [8], it is true if $A$ is the semigroup algebra $\mathbb{R}[S]$ of a commutative unital semigroup $S$. For general algebras the answer is negative, as the following example shows.

Example 7. Let $A$ be the Arens algebra $L_{\mathbb{R}}^{\omega}(0,1):=\cap_{p \geq 1} L_{\mathbb{R}}^{p}(0,1)$, where $L_{\mathbb{R}}^{p}(0,1)$ is the real $L^{p}$-space with respect to the Lebesgue measure. Then $A$ is a commutative unital real algebra which has no character (see [4] or [23, Example 2.5.10]), so that $\operatorname{Rad}_{\mathrm{ch}}(A)=A$. Let $\varphi \in C_{0}(0,1), \varphi \neq 0$. Then

$$
L_{\varphi}(f)=\int_{0}^{1} f(x)|\varphi(x)|^{2} d x, \quad f \in A,
$$

is a nonzero positive functional $L_{\varphi}$ on $A$. Obviously, it does not vanish on $\operatorname{Rad}_{\mathrm{ch}}(A)$.

Remark 8. Let $C$ be a (real or complex) unital *-algebra. The *-radical $\operatorname{Rad}(C)$ is usually defined as the ideal of $c \in C$ such that $L\left(c^{*} c\right)=0$ for all positive linear functionals $L$ on $C$, or equivalently, of elements of $C$ which are annihilated by all (possibly unbounded) *-representations of $C$.

The commutative real algebra $A$ is a $*$-algebra with identity map as involution. For such an algebra $A$ the ideal $\operatorname{Rad}_{\mathrm{ch}}(A)$ defined by (6) can be considered as another version of the radical. Then $(*)$ is the question when $\operatorname{Rad}_{\mathrm{ch}}(A)$ coincides with $\operatorname{Rad}(A)$. For the algebra $A$ in Example 7 we have $\operatorname{Rad}_{\text {ch }}(A)=A$ and $\operatorname{Rad}(A)=\{0\}$.

\section{Integral representation of linear functionals}

Now we begin with some preparations for our main result (Theorem 12).

Let $Q$ denote the unital monoid generated by the elements $1+t^{2}$, where $t \in S$, and let $B:=Q^{-1} A$ be the corresponding algebra of fractions. 
Lemma 9. Each character $\chi \in \hat{A}$ extends uniquely to a character, denoted again by $\chi$ with a slight abuse of notation, of $B=Q^{-1} A$ by setting $\chi\left(q^{-1}\right)=\chi(q)^{-1}$, $q \in Q$. The map $\widehat{B} \ni \chi \mapsto \chi\left\lceil T \in T^{*}\right.$ is injective.

Proof. Let $\chi \in \hat{A}$. Let $q \in Q, q \neq 1$. Then $q$ is of the form $q=\left(1+t_{1}^{2}\right) \ldots\left(1+t_{k}^{2}\right)$ with $t_{1}, \ldots, t_{k} \in T$ and

$$
\chi(q)=\left(1+\chi\left(t_{1}\right)^{2}\right) \ldots\left(1+\chi\left(t_{k}\right)^{2}\right)>0 .
$$

Any element $b$ of $Q^{-1} A$ is of the form $b=q^{-1} a$ with $q_{1} \in Q$ and $a \in A$. Note that $\chi(q)>0$ by $(7)$. We define $\chi(b)=\chi(q)^{-1} \chi(a)$. One easily checks that this definition is independent of the particular representation $b=q^{-1} a$ of the element $b \in B$ and that $\chi$ becomes a character on the algebra $B=Q^{-1} A$. This completes the proof of the first assertion.

Since $T$ generates the algebra $A$, each character of $A$, by the preceding also each character of $B$, is uniquely determined by its values on $T$. This implies the injectivity of the map $\chi \mapsto \chi\lceil T$.

Thus, the characters of $A$ are precisely the restriction of characters of $B$. As usual we will consider elements of $B$ as functions on the character set $\widehat{B}$ and write $b(\chi):=\chi(b)$ for $\chi \in \widehat{B}$ and $b \in B$.

The crucial technical result for our approach is the following lemma. Similar algebras of fractions have been used in [25] and also in [15].

Lemma 10. Let $\mathcal{F}$ be a finite-dimensional linear subspace of $T$ and let $\left\{t_{1}, \ldots, t_{k}\right\}$ be a vector space basis of $\mathcal{F}$. Let $B_{F}$ denote the unital subalgebra of $B$ generated by $t_{i}$ and $a_{i}:=\left(1+t_{i}^{2}\right)^{-1}$ for $i=1, \ldots, k$. For each positive linear functional $L$ on $B_{F}$ there exists a unique Radon measure $\nu_{F}$ on $\mathcal{F}^{*}$ such that

$$
L(b)=\int_{\mathcal{F}^{*}} b(\chi) d \nu_{\mathcal{F}}(\chi) \text { for } b \in B_{F} .
$$

Before we prove this lemma let us rewrite the integral (8) in terms of coordinates on the vector space $\mathcal{F}^{*}$. This builds the bridge to the moment problem on $\mathbb{R}^{k}$. Define functionals $\chi_{i} \in \mathcal{F}^{*}$ by $\chi_{i}\left(t_{j}\right)=\delta_{i j}$, where $i, j=1, \ldots, k$. Clearly, $\left\{\chi_{1}, \ldots, \chi_{k}\right\}$ is a vector space basis of $\mathcal{F}^{*}$. It is the dual basis to the basis $\left\{t_{1}, \ldots, t_{k}\right\}$ of $\mathcal{F}$. Hence each $\chi \in \mathcal{F}^{*}$ can be written as

$$
\chi=x_{1} \chi_{1}+\ldots+x_{k} \chi_{k} \quad \text { with } x(\chi):=\left(x_{1}, \ldots, x_{k}\right) \in \mathbb{R}^{k}
$$

uniquely determined by $\chi$. We shall write $\tilde{b}(x):=b(\chi)$ for $\chi \in \mathcal{F}^{*}$ and $b \in B_{F}$. Then $\tilde{b}$ is a function on $\mathbb{R}^{k}$. Let $\tilde{\nu}_{\mathcal{F}}$ denote the Radon measure on $\mathbb{R}^{k}$ defined by $\left.\tilde{\nu}_{\mathcal{F}}(x(\chi))\right):=$ $\nu_{\mathcal{F}}(\chi)$. Then we have

$$
\int_{\mathcal{F}^{*}} b(\chi) d \nu_{\mathcal{F}}(\chi)=\int_{\mathbb{R}^{k}} \tilde{b}(x) d \tilde{\nu}_{\mathcal{F}}(x) \quad \text { for } b \in B_{F} .
$$


Proof of Lemma 10. The existence assertion will be derived from the general fibre theorem proved in [26]. For $i=1, \ldots, k$, we set $b_{i}:=t_{i}\left(1+t_{i}^{2}\right)^{-1}$. Clearly, $b_{i} \in B_{F}$. Let $\chi \in \widehat{B_{F}}$. From the identity $\left(a_{i}-1 / 2\right)^{2}+b_{i}^{2}=1 / 4$ we conclude that

$$
\left(\chi\left(a_{i}\right)-1 / 2\right)^{2}+\chi\left(b_{i}\right)^{2}=1 / 4 .
$$

Therefore, $\left|\chi\left(a_{i}-1 / 2\right)\right| \leq 1 / 2$, hence $\left|\chi\left(a_{i}\right)\right| \leq 1$, and $\left|\chi\left(b_{i}\right)\right| \leq 1 / 2$. This shows that the $2 k$ functions $h_{i}=a_{i}$ and $h_{k+i}:=b_{i}$, where $i=1, \ldots, k$, on $\widehat{B_{F}}$ are bounded. We apply the fibre theorem to the bounded functions $h_{1}, \ldots, h_{2 k}$ and the preordering $\mathcal{P}:=\sum\left(B_{F}\right)^{2}$. Note that $\mathcal{K}(\mathcal{P})=\widehat{B_{F}}$.

Let us fix numbers $\lambda_{i} \in h_{i}(\mathcal{K}(\mathcal{P})), i=1, \ldots, 2 k$, and set $\lambda=\left(\lambda_{1}, \ldots, \lambda_{2 k}\right) \in \mathbb{R}^{2 k}$. For $\chi \in \widehat{B_{F}}$ and $i=1, \ldots, k$, we obtain

$$
\left.\left(1+\chi\left(t_{i}\right)^{2}\right) \chi\left(h_{i}\right)=\chi\left(1+t_{i}^{2}\right) \chi\left(a_{i}\right)=\chi\left(1+t_{i}^{2}\right) a_{i}\right)=\chi(1)=1,
$$

so that $\chi\left(h_{i}\right)>0$ and therefore $\lambda_{i}>0$. Let $\mathcal{I}_{\lambda}$ denote the ideal of $B_{F}$ generated by the functions $h_{i}-\lambda_{i}, i=1, \ldots, 2 k$. In the algebra $B_{F} / \mathcal{I}_{\lambda}$ we have $h_{i}=\left(1+t_{i}^{2}\right)^{-1}=\lambda_{i}$ and $h_{k+i}=t_{i}\left(1+t_{i}^{2}\right)^{-1}=\lambda_{k+i}$, so that $t_{i}=\lambda_{k+i} \lambda_{i}^{-1}$ for $i=1, \ldots, k$. Hence the algebra $B_{F} / \mathcal{I}_{\lambda}$ is $\mathbb{R}$, so it obviously obeys property (MP). Thus, all assumptions of the fibre theorem are satisfied. By the fibre theorem [26] (or equivalently, by the implication $($ iii $) \rightarrow\left(\right.$ i) of $\left[27\right.$, Theorem 13.10]), the preordering $\sum\left(B_{F}\right)^{2}$ of the algebra $B_{F}$ has property (MP). This means that the positive linear functional $L$ on $B_{F}$ is an integral of some Radon measure $\nu$ on $\widehat{B_{F}}$. From Lemma 9 , applied to $B_{F}$ instead of $B$, it follows that $\widehat{B_{F}} \ni \chi \rightarrow \chi\left\lceil\mathcal{F} \in \mathcal{F}^{*}\right.$ is injective. If $\nu_{\mathcal{F}}$ denotes the pushforward of the measure $\nu$ under this mapping, we obtain the desired integral representation (8).

Finally, we prove the uniqueness assertion. Let $\mu_{F}$ and $\nu_{F}$ be two Radon measures on $\mathcal{F}^{*}$ for which (8) is satisfied. Set $h_{i}:=\left(1+t_{i}^{2}\right)^{-1}$ and $h_{k+i}:=t_{i}\left(1+t_{i}^{2}\right)^{-1}$, $i=1, \ldots, k$. Let $C_{F}$ denote the subalgebra of $B_{F}$ generated all elements $h_{i_{1}} \ldots h_{i_{k}}$, where $i_{j} \in\{j, k+j\}$, and let $D_{F}$ be the algebra of functions $\tilde{h}$, where $h \in C_{F}$. Clearly, $\widetilde{h_{i}}(x)=\left(1+x_{i}^{2}\right)^{-1}$ and $\widetilde{h_{k+i}}(x)=x_{i}\left(1+x_{i}^{2}\right)^{-1}$ for $x=\left(x_{1}, \ldots, x_{k}\right) \in \mathbb{R}^{k}$ and $i=1, \ldots, k$. Further, if $h:=h_{i_{1}} \ldots h_{i_{k}}$, then $\tilde{h}=\widetilde{h_{i_{1}}} \ldots h_{i_{k}}$. This implies that all functions of $D_{F}$ vanish at infinity. Clearly, the algebra $D_{F}$ separates the points of $\mathbb{R}^{k}$. For $g:=$ $h_{1} \ldots h_{k}$ we obviously have $\tilde{g} \neq 0$ on $\mathbb{R}^{k}$. Clearly, $D_{F}$ is an algebra of continuous functions on the locally compact space $\mathbb{R}^{k}$. As shown by the preceding, this algebra satisfies the assumptions of the Stone-Weierstrass theorem (see e.g. [12, Chapter V, Corollary 8.6]). By this theorem, for each function $f \in C_{0}\left(\mathbb{R}^{k}\right)$ there exists a sequence $\left(f_{n}\right)_{n \in \mathbb{N}}$ of elements from $C_{F}$ such that the sequence $\left(\widetilde{f_{n}}\right)_{n \in \mathbb{N}}$ of $D_{F}$ converges to $f$ uniformly on $\mathbb{R}^{k}$. By (8) and (9), we have

$$
\int_{\mathbb{R}^{k}} \widetilde{f_{n}}(x) d \tilde{\mu}_{F}(x)=\int_{\mathcal{F}^{*}} f_{n}(\chi) d \mu_{\mathcal{F}}(\chi)=\int_{\mathcal{F}^{*}} f_{n}(\chi) d \nu_{F}(\chi)=\int_{\mathbb{R}^{k}} \widetilde{f_{n}}(x) d \tilde{\nu}_{F}(x) .
$$


Passing to the limit in (10) we get $\int f(x) d \tilde{\mu}_{F}=\int f(x) d \tilde{\nu}_{F}$. Since this holds for all $f \in C_{0}\left(\mathbb{R}^{k}\right)$, we conclude that $\tilde{\mu}_{F}=\tilde{\nu}_{F}$. Hence $\mu_{F}=\nu_{F}$.

Theorem 11. Let $L$ be a positive linear functional on $B$ such that $L(1)=1$. Then there exists a unique cylinder measure $\mu$ on $\mathcal{T}^{\prime}$ such that

$$
\tilde{L}(b)=\int_{\mathcal{T}^{\prime}} b(\chi) d \mu(\chi) \quad \text { for } b \in B .
$$

Proof. Let $\mathcal{F}$ be a finite-dimensional linear subspace of $\mathcal{T}$. By Lemma 10 there exists a unique Radon measure $\nu_{\mathcal{F}}$ on $\mathcal{F}^{*} \cong \mathcal{T}^{\prime} / \mathcal{F}^{\circ}$ such that (8) holds. Since $1 \in B_{F}$ and $L(1)=1$ by assumption, each measure $\nu_{\mathcal{F}}$ is normalized. If $N$ is a cylinder set with base $M$ and generating space $\mathcal{T}^{\prime} / \mathcal{F}^{\circ}$, we define $\mu(N):=\nu_{\mathcal{F}}(M)$. As noted above, to show that $\mu$ is a well-defined cylinder measure on $\mathcal{T}^{\prime}$ it suffices to verify the compatibility condition (3).

Let $\mathcal{G}$ be another finite-dimensional linear subspace of $\mathcal{T}$ such that $\mathcal{F} \subset \mathcal{G}$. We choose a basis $G:=\left\{f_{1}, \ldots, f_{k}, f_{k+1}, \ldots, f_{n}\right\}, k<n$, of the vector space $\mathcal{G}$ such that $F:=\left\{f_{1}, \ldots, f_{k}\right\}$ is a basis of $\mathcal{F}$. Let $\nu_{\mathcal{G}}$ be the corresponding measure for $\mathcal{G}$ according to Lemma 10. Further, we denote by $\sigma$ the Radon measure on $\mathbb{R}^{k}$ given by $\sigma(M)=\tilde{\nu}_{\mathcal{G}}\left(M \times \mathbb{R}^{n-k}\right)$, where $M$ is a Borel set of $\mathbb{R}^{k}$. We use the notation and some arguments from the proof of Lemma 10. Let $b \in B_{F}$. Since $F \subseteq G$, we have $b \in B_{G}$. Using (8) and (9) for both $\nu_{\mathcal{F}}$ and $\nu_{\mathcal{G}}$ we derive

$$
\begin{aligned}
\int_{\mathbb{R}^{k}} \tilde{b}(x) d \tilde{\nu}_{F}(x) & =\int_{\mathcal{F}^{*}} b(\chi) d \nu_{F}(\chi)=L(b)=\int_{\mathcal{G}^{*}} b(\chi) d \nu_{G}(\chi) \\
& =\int_{\mathbb{R}^{n}} \tilde{b}(y) d \tilde{\nu}_{G}(y)=\int_{\mathbb{R}^{k}} \tilde{b}(x) d \sigma(x) .
\end{aligned}
$$

Here the last equality follows from the fact that for $b \in B_{F}$ the function $\tilde{b}$ on $\mathbb{R}^{n}$ depends only on the first $k$ coordinates. By Lemma $10, \mu_{F}$ is the unique Radon measure on $\mathcal{F}^{*}$ satisfying (8). Hence $\tilde{\nu}_{\mathcal{F}}$ is the unique Radon measure on $\mathbb{R}^{k}$ such that $\int \tilde{b} d \tilde{\nu}_{\mathcal{F}}=L(b)$ for all $b \in B_{F}$. Therefore, it follows from (12)-(13) that $\tilde{\nu}_{\mathcal{F}}=\sigma$. Hence $\tilde{\nu}_{\mathcal{F}}(M)=\sigma(M)=\tilde{\nu}_{\mathcal{G}}\left(M \times \mathbb{R}^{n-k}\right)$ for all Borel sets $M$ of $\mathbb{R}^{k}$. It is easily verified that the latter is just condition (3) reformulated in terms of coordinates. This completes the proof of the fact that $\mu$ is a well-defined cylinder measure.

Let $b \in B$. Then $b$ is contained in some algebra $B_{F}$, so that (8) holds. Since $\mu(N):=\nu_{\mathcal{F}}(M)$, this implies that (11) holds. The uniqueness of $\mu$ follows at once from the uniqueness of the measures $\nu_{\mathcal{F}}$ stated in Lemma 10.

Now we turn to the moment problem on the algebra $A$. Define

$$
A_{+}=\{f \in A: f(\chi) \geq 0 \quad \text { for } \chi \in \hat{A}\} .
$$


Clearly, each moment functional $L$ is $A_{+}$-positive (by (5)) and satisfies $L(1)=1$ (since $\mu\left(\mathcal{T}^{\prime}\right)=1$ ) by Definition $1($ ii $)$ ).

Further, if $L$ is a positive functional on $A$, we will say that Carleman's condition holds for an element $t \in A$ if

$$
\sum_{n=1}^{\infty} L\left(t^{2 n}\right)^{-\frac{1}{2 n}}=+\infty .
$$

(Note that $L\left(t^{2 n}\right) \geq 0$, because the functional $L$ is positive.)

The main result of this paper is the following theorem.

Theorem 12. Let $L$ be a linear functional on $A$ such that $L(1)=1$. Suppose that one of the following assumptions (i) or (ii) is satisfied:

(i) $L$ is $A_{+}$-positive, that is, $L(a) \geq 0$ for $a \in A_{+}$.

(ii) $L$ is positive, that is, $L\left(a^{2}\right) \geq 0$ for $a \in A$, and Carleman's condition (14) holds for all $t$ of the vector space basis $S$ of $T$.

Then there exists a cylinder measure $\mu$ on $\mathcal{T}^{\prime}$ such that

$$
L(f)=\int_{\mathcal{T}^{\prime}} f(\chi) d \mu(\chi) \quad \text { for } f \in A .
$$

If there exists a continuous seminorm $q$ on $\mathcal{T}$ such that

$$
L\left(t^{2}\right) \leq q(t)^{2} \quad \text { for } t \in \mathcal{T}
$$

then there is a continous cylinder measure $\mu$ on $\mathcal{T}^{\prime}$ according to Definition 2 such that (15) holds.

Further, if in addition $\mathcal{T}^{\prime} \subseteq \hat{A}\lceil T$, then $L$ is a moment functional on $A$ according to Definition 5.

Proof. First assume (i). Let $B=Q^{-1} A$ the algebra of fractions defined above. Since the characters of $A$ are restrictions to $A$ of characters of $B$ by Lemma 9, we have $B_{+} \cap A=A_{+}$. Since all elements $a_{i}, b_{i}$ are bounded on $\widehat{B}$ as shown in the proof of Lemma 10, so are all elements of $Q^{-1}$. Hence for each $b \in B$ there exists $a \in A$ such that $b(\chi) \leq a(\chi)$ for all $\chi \in \widehat{B}$. Therefore, the $A_{+}$-positive functional $L$ can be extended to a $B_{+}$-positive linear functional $\tilde{L}$ on $B$. From Theorem 11 , applied to $\tilde{L}$, it follows that there exists a cylinder measure $\mu$ on $\mathcal{T}^{\prime}$ such that (15) is satisfied.

Now we suppose that (ii) is satisfied. Let $\mathcal{F}$ be a finite-dimensional linear subspace of $T$ and $F:=\left\{f_{1}, \ldots, f_{k}\right\}$ a basis of $\mathcal{F}$. Let $A_{F}$ denote the unital subalgebra of $A$ generated by $f_{1}, \ldots, f_{k}$. We define a linear functional $L_{F}$ on $\mathbb{R}\left[x_{1}, \ldots, x_{k}\right]$ by

$$
L_{F}\left(p\left(x_{1}, \ldots, x_{k}\right)\right)=L\left(p\left(f_{1}, \ldots, f_{k}\right)\right) .
$$


Since $L_{F}\left(x_{j}^{2 n}\right)=L\left(f_{j}^{2 n}\right)$ for $j=1, \ldots, k$ and $n \in \mathbb{N}$, it follows from (ii) that $L_{F}$ is a positive functional on $\mathbb{R}\left[x_{1}, \ldots, x_{k}\right]$ satisfying the multi-variate Carleman condition. Therefore, by Nussbaum's theorem, $L_{F}$ is a determinate moment functional on $\mathbb{R}\left[x_{1}, \ldots, x_{k}\right]$, that is, $L_{F}$ has a unique representing Radon measure on $\mathbb{R}^{k}$. Therefore, there exists a unique Radon measure $\nu_{\mathcal{F}}$ on $\mathcal{F}^{*} \cong \mathbb{R}^{k}$ such that

$$
L(a)=\int_{\mathcal{F}^{*}} a(\chi) d \nu_{\mathcal{F}}(\chi) \quad \text { for } a \in A_{F} .
$$

Proceeding as in the proof of Theorem 11 it follows that the family of measures $\nu_{F}$ give a well-defined cylinder measure on $\mathcal{T}^{\prime}$. The uniqueness of measures $\nu_{F}$ used in a crucial manner in the proof of Theorem 11 follows now from the uniqueness of representing measures of functionals $L_{F}$ because of the Carleman condition.

Now suppose that the continuity assumption (16) is fulfilled. Let $\varepsilon>0$ and $a>0$ be given. Set $\delta:=a \sqrt{\varepsilon}$ and $U:=\{t \in T: q(t) \leq \delta\}$. Then, for $t \in U$,

$$
\begin{aligned}
& \mu\left(\left\{\chi \in \mathcal{T}^{\prime}:|\chi(t)| \geq 1\right\}\right) \leq a^{-2} \int_{\mathcal{T}^{\prime}} \chi(t)^{2} d \mu(\chi) \\
& \quad=a^{-2} \int_{\mathcal{T}^{\prime}} \chi\left(t^{2}\right) d \mu(\chi)=a^{-2} L\left(t^{2}\right) \leq a^{-2} q(t)^{2} \leq a^{-2} \delta^{2}=\varepsilon
\end{aligned}
$$

Thus, the condition in Definition 2 is satisfied, so $\mu$ is continuous.

If $\mathcal{T}^{\prime} \subseteq \hat{A}\lceil T$, then Definition 5 is satisfied, so $L$ is a moment functional on $A$.

\section{Nuclearity assumptions and representing measures}

If we assume the nuclearity of the space $\mathcal{T}$ in Theorem 12, then we get an "ordinary" $\sigma$-additive representing measure of $L$ rather than a cylinder measure.

Theorem 13. Let $L$ be a linear functional on $A$ such that one of the assumptions (i) or (ii) in Theorem 12 holds. Suppose that $\mathcal{T}$ is a nuclear locally convex Hausdorff space and there exists a continuous seminorm $q$ on $\mathcal{T}$ such that

$$
L\left(t^{2}\right) \leq q(t)^{2} \quad \text { for } \quad t \in \mathcal{T}
$$

Then there exists a measure $\mu$ defined on the $\sigma$-algebra $\mathcal{B}(\mathcal{T})$ such that

$$
L(f)=\int_{\mathcal{T}^{\prime}} f(\chi) d \mu(\chi) \text { for } f \in A .
$$

If $\mathcal{T}^{\prime} \subseteq \hat{A}\lceil T$, then $L$ is a moment functional with representing measure $\mu$. 
Proof. Assumption (i) implies that $L$ is positive. Therefore, if $L(1)=0$, then $L=0$ by the Cauchy-Schwarz inequality, so the assertion holds trivially with $\mu=0$.

Suppose that $L(1) \neq 0$. Then $L(1)>0$ by (i) or (ii) and Theorem 12 applies to the functional $L(1)^{-1} L$ on $A$. Since the space $\mathcal{T}$ is nuclear, the corresponding cylinder measure is a measure by Minlos' Theorem 3 .

By Minlos theorem, the nuclearity of the space $\mathcal{T}$ implies that each cylinder measure on $\mathcal{T}^{\prime}$ is indeed a measure. A necessary and sufficient condition when a given cylinder measure is a measure is provided by Sazonov's Theorem 4. We do not restate the corresponding result in the present setting.

As an important special case we can take the whole algebra $A$ as vector space $T$. Then, $\hat{A}=\hat{A}\left\lceil T\right.$, so the assumption $\mathcal{T}^{\prime} \subseteq \hat{A}\lceil T$ is trivially satisfied and Theorems 12 and 13 provide integral representations of the corresponding functional $L$ over characters.

Now we begin with the preparations for the next main result (Theorem 15).

A real algebra $C$ equipped with a locally convex topology is called a topological algebra if for each $b \in C$ the mappings $c \rightarrow b c$ and $c \rightarrow c b$ are continuous on $C$.

A locally convex space $V$ is called barrelled if each absolutely convex closed absorbing subset of $V$ is a zero neighbourhood [22, Chapter II, Section 7]. For instance, each complete metrizable locally convex space is barrelled.

The following lemma is a known result (see e.g. [23, Corollary 3.6.]) adapted to the present context.

Lemma 14. Suppose that $C$ is a barrelled topological real algebra. If $L$ is a continuous positive linear functional on $C$, there exists a continuous seminorm $q$ on $C$ such that $L\left(c^{2}\right) \leq q(c)^{2}$ for all $c \in C$.

Proof. First, we prove the equality

$$
L\left(c^{2}\right)^{1 / 2}=\sup \left\{|L(c b)|: b \in C ; L\left(b^{2}\right)=1\right\} .
$$

Let $M_{c}$ denote the supremum on the right hand side of (19). Since $L$ is positive, the Cauchy-Schwarz inequality yields $|L(c b)| \leq L\left(c^{2}\right)^{1 / 2} L\left(b^{2}\right)^{1 / 2}=L\left(c^{2}\right)^{1 / 2}$. Hence $M_{c} \leq L\left(c^{2}\right)^{1 / 2}$. Further, if $L\left(c^{2}\right)=0$, then $M_{c}=0$ by this equality. Now suppose that $L\left(c^{2}\right)>0$. Then $b:=c L\left(c^{2}\right)^{-1 / 2}$ satisfies $L\left(b^{2}\right)=1$ and $|L(c b)|=L\left(c^{2}\right)^{1 / 2}$, so that $L\left(c^{2}\right)^{1 / 2} \leq M_{c}$. Putting the preceding together we have proved (19).

Now we set $U:=\left\{c \in C: L\left(c^{2}\right)^{1 / 2} \leq 1\right\}$. Obviously, $U$ is absorbing. From (19) we easily conclude that the set $U$ is absolutely convex.

We prove that $U$ is closed in $C$. Let $\left(c_{i}\right)_{i \in I}$ be a net from $U$ converging to some element $c \in C$. Fix $b \in C$ such that $L\left(b^{2}\right)=1$. Since $L$ is continuous and the map $a \rightarrow a b$ on $C$ is also continuous (because $A$ is a topological algebra), we 
conclude that $\lim _{i} L\left(c_{i} b\right)=L(c b)$. From $c_{i} \in U$ and (19) it follows that $\left|L\left(c_{i} b\right)\right| \leq 1$, so that $|L(c b)| \leq 1$. Therefore, again by (19), $L\left(c^{2}\right)^{1 / 2} \leq 1$, that is, $c \in U$. This shows that $U$ is closed.

Since the locally convex space $C$ is barrelled, $U$ is a neighbourhood of zero in $C$. Hence there exists a continuous seminorm $q$ on $C$ such that $\{c \in C: q(c) \leq 1\} \subseteq U$. This implies that $L\left(c^{2}\right)^{1 / 2} \leq q(c)$ for all $c \in C$. (In fact, $r(c):=L\left(c^{2}\right)^{1 / 2}, c \in C$, defines a seminorm $r$ on $C$. Hence $r$ is continuous on $C$ and we could take $r=q$.) [9].

Under assumption (i) the next result was proved by Borchers and Yngavson

Theorem 15. Suppose that the commutative real unital algebra $A$ carries a nuclear barrelled locally convex covex topology such that it is a topological algebra. Let $L$ be a continuous linear functional on A satisfying one of the following assumptions:

(i) $L$ is $A_{+}$-positive.

(ii) $L$ is positive and Carleman's condition (14) holds for all $t$ of some vector space basis of $A$.

Then there exists a measure $\mu$ on the $\sigma$-algebra $\mathcal{B}(\hat{A})$ such that

$$
L(a)=\int_{\hat{A}} a(\chi) d \mu(\chi) \text { for } a \in A .
$$

Proof. Since $A$ is a barrelled topological algebra, by Lemma 14 there is a continuous seminorm $q$ on $A$ such that $L\left(a^{2}\right) \leq q(a)^{2}$ for $a \in A$. Therefore, setting $T=A$, all assumptions of Theorem 13 are fulfilled and the assertion follows from Theorem 13.

\section{Moment problem for symmetric algebras over vector spaces}

Let $V$ be a real vector space. First we recall the definition of the symmetric algebra $S(V)$ over $V$.

For $n \in \mathbb{N}, V_{n}=V \otimes \ldots \otimes V$ denotes the $n$-fold tensor product of $V$ and $S_{n}(V)$ is the subspace of symmetric tensors. For $v=\sum_{i} v_{1 i} \otimes \ldots \otimes v_{n i} \in V_{n}$ we set

$$
s(v):=\frac{1}{n !} \sum_{\theta} \sum_{i} v_{\theta(1) i} \otimes \ldots \otimes v_{\theta(n) i},
$$

where the summation is over all permutation $\theta$ of $\{1, \ldots, n\}$. Set $S_{1}(V):=V$ and $S_{0}(V):=\mathbb{R}$. Let $S(V)$ be the direct sum of vector spaces $S_{n}(V), n \in \mathbb{N}_{0}$. Then $S(V)$ becomes a commutative unital real algebra with product determined by

$$
(v \cdot w)_{m}=\sum_{k+n=m} s\left(v_{k} \otimes w_{n}\right) \quad \text { for } v=\left(v_{k}\right), w=\left(w_{k}\right) \in S(V)
$$


with the obvious interpretations $v_{0} \otimes w_{n}=v_{0} w_{n}, v_{n} \otimes w_{0}=w_{n} v_{0}, s\left(v_{0} \otimes w_{0}\right)=v_{0} w_{0}$ and the unit element $1=(1,0,0, \ldots)$. That is, $S(V)$ is the free commutative real unital algebra over the vector space $V$; it is called the symmetric algebra over $V$.

Let $V^{*}$ be the dual vector space of $V$. Since $S(V)$ is the free commutative algebra over $V$, each functional $L \in V^{*}$ extends uniquely to a character of $S(V)$. Hence the restriction $L \rightarrow L\left\lceil V\right.$ is a bijection of $\widehat{S(V)}$ and $V^{*}$. That is, the linear functionals on $V$ are in one-to-one correspondence with characters of the algebra $S(V)$. Letting $A=S(V)$ and $T=V$, we are in the setup described at the beginning of Section 3 and we have $\hat{A}\left\lceil T=V^{*}\right.$.

The second assertion of the next theorem is the well-known solution of the moment problem over nuclear spaces. It was first obtained independently in [9] and [11] by using different methods. An elegant approach based on Choquet theory was sketched in [17] and elaborated in [23, Section 15.1].

Theorem 16. Let $V$ be a real vector space and let $L$ be a linear functional on $S(V)$ such that $L(1)=1$. Suppose one of the assumptions (i) or (ii) in Theorem 12 is satisfied. Then $L$ is a moment functional (as in Definition 5 with $\mathcal{T}^{\prime}=V^{*}$ ), so there exists a cylinder measure $\mu$ on $V^{*}$ such that

$$
L(f)=\int_{V^{*}} f(\chi) d \mu(\chi) \text { for } f \in S(V) .
$$

Suppose in addition that $V$ is equipped with a nuclear locally convex Hausdorff topology and there exists a continuous seminorm $q$ on $V$ such that

$$
L\left(t^{2}\right) \leq q(t)^{2} \quad \text { for } t \in V .
$$

Then there exists a measure $\mu$ on the $\sigma$-algebra $\mathcal{B}\left(V^{\prime}\right)$ of $V^{\prime}$ such that

$$
L(f)=\int_{V^{\prime}} f(\chi) d \mu(\chi) \quad \text { for } f \in S(V) .
$$

Proof. For the first assertion we equip the vector space $V$ with its finest locally convex topology. Then $\mathcal{T}^{\prime}=V^{*}=\hat{A}\lceil T$, so $L$ is a moment functional by the first assertion of Theorem 12.

If $V$ is a nuclear space and (22) holds, then $\mathcal{T}^{\prime}=V^{\prime} \subseteq \hat{A}\lceil T$ and the second assertion of Theorem 12 applies.

Example 17. Let $V$ be a vector space with a countable Hamel basis. Then $S(V)$ is (isomorphic to) the polynomial algebra $\mathbb{R}\left[x_{1}, \ldots, x_{n}, \ldots\right]$ in countably many indeterminants $x_{n}, n \in \mathbb{N}$. Further, the finest locally convex topology on $V$ is nuclear and each seminorm (in particular, the seminorm $t \rightarrow L\left(t^{2}\right)^{1 / 2}$ ) is continuous in this topology. Thus, Theorem 16 applies, hence each $S(V)_{+}$-positive linear functional on $S(V)$ is a moment functional with representing measure on $\mathcal{B}\left(V^{\prime}\right)$. In a slightly different formulation this result was proved in [15]. 


\section{Gaussian measures}

Let us begin with the finite-dimensional case. Let $(\cdot, \cdot)$ be a scalar product on $\mathbb{R}^{d}$ and let $d x$ be the Lebesgue measure on $\mathbb{R}^{d}$ for this scalar product. For a Borel subset $M$ of $\mathbb{R}^{d}$ we define

$$
\nu(M)=(2 \pi)^{-d / 2} \int_{M} e^{-(x, x) / 2} d x .
$$

Then $\nu$ is a Radon measure on $\mathbb{R}^{d}$, called the (standard) Gaussian measure associated with $(\cdot, \cdot)$. It can be nicely characterized in terms of its Fourier transform: $\nu$ is the unique probability Radon measure on $\mathbb{R}^{d}$ satisfying

$$
\int_{\mathbb{R}^{d}} e^{\mathrm{i}(x, y)} d \nu(x)=e^{-(y, y) / 2} \quad \text { for } y \in \mathbb{R}^{d} .
$$

Obviously, $\int_{\mathbb{R}^{d}} p(x) d \nu(x)$ is finite for each polynomial $p \in \mathbb{R}\left[x_{1}, \ldots, x_{d}\right]$.

Now we suppose that $(\cdot, \cdot)$ is a scalar product on a locally convex space $V$.

Let $\mathcal{F}$ be a finite-dimensional subspace of $V$. We identify $\mathcal{F}$ with some $\mathbb{R}^{d}$ by choosing a basis in $\mathcal{F}$ which is orthonormal with respect to the scalar product $(\cdot, \cdot)$. Let $\nu_{\mathcal{F}}$ denote the Gaussian measure on $\mathcal{F}$ associated with the restriction of the scalar product $(\cdot, \cdot)$ to $\mathcal{F}$. The finite-dimensional Hilbert space $(\mathcal{F},(\cdot, \cdot))$ is canonically isomorphic to its dual and so to $V^{\prime} / \mathcal{F}^{\circ}$. Let $I_{\mathcal{F}}$ denote this isomorphism. Then $\mu_{\mathcal{F}}(\cdot):=\nu_{\mathcal{F}}\left(I_{\mathcal{F}}^{-1}(\cdot)\right)$ defines a Radon measure on $V^{\prime} / \mathcal{F}^{\circ}$. These measures $\mu_{\mathcal{F}}$ satisfy the compatibility condition (3), so they define a cylinder measure $\mu:=\mu_{(\cdot, \cdot)}$ on $V^{\prime}$, called the Gaussian cylinder measure associated with the scalar product $(\cdot, \cdot)$.

If the scalar product $(\cdot, \cdot)$ is continuous on $V \times V$, it follows easily that $\mu_{(\cdot, \cdot)}$ satisfies the continuity condition in Definition $(2)$, so $\mu_{(\cdot, \cdot)}$ is continuous. Details and proofs for the preceding construction can be found e.g. in [16, Ch. IV, §3].

Theorem 18. Let $V$ be a real locally convex Hausdorff space. Suppose that $(\cdot, \cdot)$ is a scalar product on $V$ which is continuous on $V \times V$. Then $\mu_{(\cdot, \cdot)}$ is a continuous cylinder measure on $V^{\prime}$ and there exist a determinate moment functional $L$ on $A=S(V)$ such that

$$
L(f)=\int_{\mathcal{T}^{\prime}} f(\chi) d \mu_{(\cdot, \cdot)}(\chi) \quad \text { for } f \in S(V) .
$$

If the locally convex space $E$ is nuclear, then $\mu_{(\cdot, \cdot)}$ is measure on $\mathcal{B}\left(V^{\prime}\right)$.

Proof. Since for Gaussian measures on finite-dimensional spaces integrals of polynomials are finite, the integrals in (25) are finite. Hence equation (25) gives a well-defined moment functional $L$ on $A$. 
Let $\tau$ be a representing cylinder measure for the moment functional $L$. Then for each finite-dimensional subspace $\mathcal{F}$ of $E, \tau_{\mathcal{F}}$ and $\mu_{\mathcal{F}}$ have the same moment functional on $E^{\prime} / \mathcal{F}^{\circ}$ and so have their push forwards on $\mathcal{F}$. But $\nu_{\mathcal{F}}$ (as defined above) is a Gaussian measure on $\mathbb{R}^{d}$. It it well-known and follows (for instance) from [27, Corollary 14.24] that $\nu_{\mathcal{F}}$ is determinate. Therefore, both push forwards coincide on $\mathcal{F}$. Hence $\tau_{\mathcal{F}}=\mu_{\mathcal{F}}$ which implies that $\tau=\mu_{(\cdot, \cdot)}$. This shows that $L$ has a unique representing cylinder measure $\mu_{(\cdot, \cdot)}$, that is, $L$ is determinate.

If the space $E$ is nuclear, the cylinder measure $\mu_{(\cdot, \cdot)}$ is indeed a measure by Minlos' Theorem 3.

Further, the Gaussian cylinder measure $\mu_{(\cdot, \cdot)}$ yields a positive definite functions $\widehat{\mu(\cdot, \cdot)}$ on $V$, called the Fourier transform of $\mu_{(\cdot, \cdot)}$, defined by

$$
\widehat{\mu_{(\cdot, \cdot)}}(y)=\int_{V^{\prime}} e^{\mathrm{i}(\chi, y)} d \mu_{(\cdot, \cdot)}(\chi), \quad y \in V .
$$

Cylinder measures are often studied in terms of their Fourier transforms and there is a Bochner-Minlos theorem, see e.g. [16, Chapter IV, §4].

Now we specialize to the case where $V$ is a real separable Hilbert space with scalar product $\langle\cdot, \cdot\rangle$ and assume that the scalar product $(\cdot, \cdot)$ is continuous on $V$. From the latter it follows that there exists a bounded positive self-adjoint operator $b$ on $V$ such that ker $b=\{0\}$ and

$$
(x, y)=\langle b x, y\rangle, \quad x, y \in V .
$$

Conversely, each positive bounded operator $b$ on $V$ with trivial kernel defines a continuous scalar product on $E$ by $(27)$.

The following result characterizes when the cylinder measure $\mu_{(\cdot, \cdot)}$ is a measure.

Proposition 19. The Gaussian cylinder measure $\mu_{(\cdot, \cdot)}$ on $V^{\prime}$ defined above yields a measure on the $\sigma$-algebra $\mathcal{B}\left(V^{\prime}\right)$ if and only if the operator $b$ is trace class.

Proof. [16, Hilfssatz 2, p. 313] or [13, Chapter II, Theorem 2.1].

Example 20. Suppose that $\left\{e_{k} ; k \in \mathbb{N}\right\}$ is an orthornormal basis of a Hilbert space $V$ and let $\left(b_{k}\right)_{k \in \mathbb{N}}$ a bounded sequence of positive numbers. There is a unique bounded operator $b$ on $V$ defined by $b e_{k}=b_{k} e_{k}$ for $k \in \mathbb{N}$. Obviously, $b$ is positive, self-adjoint and ker $b=\{0\}$. Let $(\cdot, \cdot)$ denote the scalar product $(27)$ on $V$ and $\mu_{(\cdot, \cdot)}$ the corresponding Gaussian cylinder measure on $V^{\prime} \cong V$. By Theorem 18, $\mu_{(\cdot, \cdot)}$ is the unique representing cylinder measure for the moment functional $L$ (defined by (25)) on $S(V)$. 
Clearly, $b$ is a trace class operator if and only if

$$
\sum_{k=1}^{\infty} b_{k}<+\infty .
$$

Therefore, by Proposition 19, the moment functional $L$ has a representing ( $\sigma$-additive!) measure on $\mathcal{B}\left(V^{\prime}\right)$ if and only if (28) holds. Thus, if $\sum_{k} b_{k}=+\infty$, then the unique representing cylinder measure of $L$ is not a measure.

\section{References}

1. Alpay, D., Jorgensen, P. E. T. and Kimsey, D. P., Moment problems in an infinite number of variables, Infin. Dimens. Anal. Quantum Probab. Relat. Top. 18 (2015), 1550024.

2. Akhiezer, N. I., The Classical Moment Problem, Oliver and Boyd, Edinburgh, 1965.

3. Albeverio, S. and Herzberg, F., The moment problem on the Wiener space, Bull. Sci. Math. 132 (2008), 7-18.

4. Arens, R., The algebra $L^{\omega}$ and convex topological rings, Bull. Amer. Math. Soc. 52 (1946), 931-935.

5. Berezansky, Y. M. and Kondratiev, Y. G., Spectral Methods in InfiniteDimensional Analysis 2, Kluwer Acad. Publishers, Dordrecht, 1995.

6. Berezansky, Y. M. and Sifrin, S. N., The generalized degree symmetric moment problem, Ukr. Mat. Zh. 23 (1971), 291-306.

7. Berg, C., Christensen, J. P. R. and Ressel, P., Harmonic Analysis on Semigroups, Graduate Texts, Springer, New York, 1984.

8. Bisgandd, T. M., Positive linear forms vanish on the radical, Positivity 4 (2000), $115-118$.

9. Borchers, H. J. and Yngvason, J., Integral representations for Schwinger functionals and the moment problem over nuclear spaces, Comm. Math. Phys. 43 (1975), 255-271.

10. Borchers, H. J. and Yngvason, J., Necessary and sufficient conditions for integral representations of Wightman functionals at Schwinger points, Comm. Math. Phys. 47 (1976), 197-214.

11. Challifour, J. L. and Slinker, S. P., Euclidean field theory I. The moment problem, Comm. Math. Phys. 43 (1975), 41-58.

12. Conway, J. B., A Course in Functional Analysis, Graduate Texts, Springer, New York, 1990.

13. Dalecky, Yu. L. and Fomin, S. V., Measures and Differential Equations in InfiniteDimensional Space, Kluwer Acad. Publishers, Dordrecht, 1991.

14. Gashemi, M. and Kuhlmann, S., Closure of the cone of sums of $2 d$-powers in commutative real topological algebras, J. Funct. Anal. 264 (2013), 413-427.

15. Gashemi, M., Kuhlmann, S. and Marshall, M., Moment problem in infinitely many variables, Israel J. Math. 212 (2016), 989-1012.

16. Gelfand, I. M. and Wilenkin, N. J., Verallgemeinerte Funktionen (Distributionen), DVW, Berlin, 1964. 
17. Hegerfeldt, G. C., Extremal decomposition of Wightman functionals and of states on nuclear *-algebras by Choquet theory, Comm. Math. Phys. 45 (1975), 133145.

18. Kostyuchenko, A. G. and Mityagin, B. S., Positive definite functionals on nuclear spaces, Tr. Mosk. Mat. Obs. 9 (1960), 283-316.

19. Infusino, M., Kuna, T. and Rota, A., The full infinite dimensional moment problem on semi-algebraic sets of generalized functions, J. Funct. Anal. 267 (2014), $1382-1418$.

20. Minlos, R. A., Generalized random processes and their extension to measures, $\operatorname{Tr}$. Mosk. Mat. Obs. 8 (1959), 497-518 (Russian).

21. Sazonov, V., A remark on characteristic functionals, Teor. Veroyatn. Primen. 3 (1958), 201-205.

22. SchÄFER, H., Topological Vector Spaces, Springer, Berlin, 1972.

23. SсhмüDgen, K., Unbounded Operator Algebras and Representation Theory, Birkhäuser-Verlag, Basel, 1990.

24. Schmüdgen, K., The $K$-moment problem for compact semi-algebraic sets, Math. Ann. 289 (1991), 203-206.

25. Schmüdgen, K., Algebras of fractions and strict Positivstellensätze for $*$-algebras, $J$. Reine Angew. Math. 647 (2010), 57-86.

26. Schmüdgen, K., A fibre theorem for moments problems and some applications, Israel J. Math. 28 (2016), 43-66.

27. Schmüdgen, K., The Moment Problem, Graduate Texts, Springer, New York, 2017.

28. Sifrin, S. N., Infinite-dimensional symmetric analogues of the Stieltjes moment problem, Ukr. Mat. Zh. 26 (1974), 69291-306.

29. Umemura, Y., Measures on infinite dimensional spaces, Publ. Res. Inst. Math. Sci. 1 (1965), 1-47.

Konrad Schmüdgen

Mathematisches Institut

Universität Leipzig

Augustusplatz 10/11

D-04109 Leipzig

Germany

schmuedgen@math.uni-leipzig.de

Received December 10, 2017

in revised form April 4, 2018 